\begin{abstract}
MS17 O1
Structural reaction of PZT under in situ conditions using Synchrotron powder diffraction - influence and stability of nanostructures Hartmut Fuess ${ }^{\mathrm{a}}$, Kristin A. Schoenau ${ }^{a}$, Ljubomira A Schmitt a, Michael Knapp , Matteo Leoni ${ }^{\mathrm{c}}$, Mario Maglione ${ }^{\mathrm{d}}{ }^{\mathrm{a}}$ Materials Science, Darmstadt University of Technology, Germany. ${ }^{\mathrm{b}} C E L L S$, Barcelona, Spain. ${ }^{c}$ Dept. Materials Engineering and Industrial Technologies, University of Trento, Italy. ${ }^{d}$ ICMCB-CNRS, Universite Bordeaux 1, France.

E-mail: hfuess@tu-darmstadt.de
\end{abstract}

Keywords: ferroelectric oxids, in situ synchrotron $x$ ray powder diffraction, phase stability, nanodomains,

Ferroelectric lead zirconate titanate, $\mathrm{PbZr}_{1-\mathrm{x}} \mathrm{Ti}_{\mathrm{x}} \mathrm{O}_{3}(\mathrm{PZT})$, is currently used in a wide range of applications converting electrical into mechanical energy. Highest strains and piezoelectric properties are found at the socalled morphotropic phase boundary (MPB) between tetragonal and rhombohedral symmetry. Previous results describe the structure at the MPB as monoclinic [1], whereas our recent studies using high-resolution synchrotron $\mathrm{x}$-ray powder diffraction in combination with TEM and EPR [2, 3] were able to correlate XRD observation with a nanodomain structure. The internal symmetry of the nanodomains is difficult to determine due to strong coherence effects in diffraction experiments, which is in correspondence with findings for relaxor ceramics using martensitic theory [4]. The stability field of these nanodomain structures is strongly dependent on sample composition, temperature and electric field. In situ synchrotron diffraction experiments in transmission mode at the beamline B2, Hasylab, Hamburg, reveal changes related to these three parameters. Furthermore, the microstructural reaction of the material is investigated under operating conditions. While the domain structure of samples with low nanodomain content is changed under electric field into that of the adjacent tetragonal or rhombohedral phase field, samples with a distinct balance between $\mathrm{Zr}$-content and tetragonal c/a-ratio show stable nanodomains. These samples undergo reversible phase transitions between a mixture of tetragonal microdomains and nanodomains at the coercive field and rhombohedral microdomains at high voltage. In-situ electric field diffraction above the transition temperature between nanodomain structures and the tetragonal phase show tetragonal domain switching. Furthermore, temperature dependent measurements of the dielectric constant are able to clarify the character of the ferroelectric-to-paraelectric transition and gives new information on the behavior of nanodomain structures under field. This enables us to correlate properties of this material with the relaxor systems PMN-PT and PZN-PT.

[1] Noheda, B; Gonzalo, J.A.; Cross, L.E.; Guo, R.; Park, S.E.; Cox, D.E.; Shirane, G. Phys. Rev. B, 2000, 61, 8687

[2] Schmitt, L.A., Schönau, K.A.; Theissmann, R.; Fuess, H.; Kungl, H.; Hoffmann, M.J.; J. Appl. Phys. 2007, 101(6), in press. [3] Schönau, K.A.; Schmitt, L.A.; Knapp, M.; Fuess, H.; Eichel, R.-A.; Kungl, H.; Hoffmann, M.J. Phys Rev B, 2007, 75(14) in press.

[4] Jin, Y.M; Wang, Y.U.; Khachaturyan, A.G.; Li, J.F.; Viehland, D.; J. Appl. Phys., 2003, 94 (5), 3629

\begin{abstract}
MS17 O2
Insights into thermoelectric candidate materials from powder diffraction. Birgitte L. Pedersen ${ }^{\mathrm{a}}$, Henrik Birkedal $^{\mathrm{a}}$, Eiji Nishibori ${ }^{\mathrm{b}}$, Poul Toft Frederiksen ${ }^{\mathrm{c}}$, Bo Brummerstedt Iversen ${ }^{\mathrm{a}}$. ${ }^{\mathrm{a}}$ Department of Chemistry \& Interdisciplinary Nanoscience center, University of Aahus, Denmark. ${ }^{\mathrm{b}}$ Department of Applied Physics, Nagoya University, Japan. Grundfos A/S, Bjerringbro, Denmark.

E-mail: blp@chem.au.dk
\end{abstract}

Keywords: synchrotron powder diffraction, thermoelectric materials, thermal decomposition

The $\beta$-phase of p-type semiconductor $\mathrm{Zn}_{4} \mathrm{Sb}_{3}$ has attracted attention because it is a potential candidate for thermoelectric purposes in the intermediate temperature range $(473-673 \mathrm{~K})[1,2]$. The thermoelectric Figure of Merit is defined as $Z T=T S^{2} \sigma / \kappa$, where $S$ is the Seebeck coefficient, $\sigma$ is the electrical conductivity, $\kappa$ is the thermal conductivity and $T$ is the absolute temperature Although $\mathrm{Zn}_{4} \mathrm{Sb}_{3}$ exists in at least three crystalline phases, only the $\beta$-phase, stable from 263-765 K, has high thermoelectric performance [1-3]. The origin of the outstanding properties is the presence of interstitial zinc sites creating a highly defect crystal structure, which effectively lowers thermal conductivity, while preserving electronic conduction $[4,5]$. Below $263 \mathrm{~K}, \mathrm{Zn}_{4} \mathrm{Sb}_{3}$ undergoes two phase transitions to $\mathrm{Zn}$-ordered highly complicated phases [6]. The combination of interstitial disorder and highly complex low temperature phases makes the crystallographic characterization of these materials challenging.

To become commercially applicable, it is crucial that the material is compactable and stable during thermal cycling and that it has the highest possible thermoelectric performance. Previous studies have shown that ZT can be improved by doping with Cd.[7] To understand and optimize the effect of doping, knowledge about the dopant atom siting is essential. We present and discuss the use of powder diffraction for the analysis of these complicated materials that represent true characterization challenges. Sample compaction, a key processing step for the use of these microcrystalline materials, may change the purity and also influence the performance of the materials [8]. As even small levels of impurities can have a profound effect on performance, high quality powder diffraction data a crucial.

We discuss the question of dopant atom location by using high resolution synchrotron powder diffraction on a slightly mercury doped sample, $\mathrm{Hg}_{0.04} \mathrm{Zn}_{3.96} \mathrm{Sb}_{3}$. Thermal stability and decomposition pattern has been studied on a pure and a Te-doped sample by multi temperature synchrotron powder diffraction. Te-doping clearly changes the decomposition pattern, and although no immediate thermal stabilization was achieved, the study suggests that doping might be a way to stabilize the system.

[1]. T. Caillat, J.-P. Fleurial, A. Borshchevsky, J. Phys. Chem. Solids, 1996, 58, 1119-1125

[2.] T. Caillat, A. Borshchevsky and J.-P. Fleurial, US Patent 6,942,728 B2, 2005.

[3.]Y. Mosharivskyj, A. O. Pecharsky, S. Bud'ko, G. J. Miller, Chem. Mater., 2004, 16, 1580-1589.

[4.]G. J. Snyder, M. Christensen, E. Nishibori, T. Caillat, B. B. Iversen, Nature Materials, 2004, 3, 458-463.

[5.] F. Caglioni, E. Nishibori, P. Rabiller, L. Bertini, M. Christensen, G. J. Snyder, C. Gatti, B. B. Iversen,. Chem. Eur. J., 
2004, 10, 3861-3870. [6] J. Nylen, S. Lidin, M. Andersson, B. B. Iversen, H. Lui, N. Newman, U. Haussermann, Chem. Mater., 2007, 19(4), 834-838.

[7] T. Caillat, J.-P. Fleurial, A. Borshchevsky, Nasatech Briefs, Feb 1999. [8] B. L. Pedersen, H. Birkedal, M. Nygren, P. T. Frederiksen, B. B. Iversen, Appl. Phys. Letters, 2006, 89(24), 242108 .

\section{MS17 O3}

Positive Electrode Materials Based On Moroccan Mineral Resources (Co, Mn, Ni) : Structural And Electrochemical Properties I. Saadoune ${ }^{1,} \mathrm{M}$. Aklalouch $^{1}$, M. Dahbi ${ }^{1}$, J.M. Amarilla ${ }^{2}, \mathrm{~K}$. Edstrom $^{3}$ And T. Gustafsson ${ }^{3}{ }^{1}$ ECME, LP2E2M, Université Cadi Ayyad, Morocco. ${ }^{2}$ ICMM CSIC, Madrid, Spain. 3 Angström Lab., Uppsala University, Sweden

\section{Keywords: Rietveld Refinement, Functionally graded Materials, Energy storage}

Intense research is worldwide devoted to the synthesis and characterization of positive electrode materials for lithium ion batteries owing to their high energy density and excellent power performances. Most of the efforts are concentrated on the two-dimensional layer-structure $\mathrm{LiMO}_{2}(\mathrm{M}: \mathrm{Co}, \mathrm{Mn}, \mathrm{Ni})$, the three-dimensional $\mathrm{LiMn}_{2} \mathrm{O}_{4}$ spinel and the $\mathrm{LiFePO}_{4}$ olivine type structure. Fortunately, Morocco produces cobalt, manganese, nickel and is the main phosphate producer in the world. For the layered $\mathrm{LiMO}_{2}$ oxide, $\mathrm{Li}^{+}$and $\mathrm{M}^{3+}$ cations are supposed to be orderly arranged along the (111) direction of the rock salt cubic lattice leading to a 2D layer structure, isostructural with $\alpha-\mathrm{NaFeO}_{2}$ (space group $\mathrm{R}-3 \mathrm{~m}$ ). $\mathrm{Li}^{+}$cations reside at Wyckoff $3 \mathrm{a}\left(\begin{array}{lll}0 & 0 & 0\end{array}\right)$ octahedral sites, $\mathrm{Ni}^{3+}$ cations are located in octahedral $3 b\left(\begin{array}{lll}0 & 0 & 1 / 2\end{array}\right)$ and oxygen anions are in a cubic close-packing, occupying the $6 \mathrm{c}\left(\begin{array}{lll}0 & 0 \mathrm{z}\end{array}\right)$ sites. Stoichiometric $\mathrm{LiNiO}_{2}(\mathrm{Li} / \mathrm{Ni}=1)$ is difficult to synthesize. Some extra-nickel ions are present in the $\mathrm{Li}$ plane leading to $\left[\mathrm{Li}_{1-\mathrm{z}} \mathrm{Ni}_{\mathrm{z}}\right]_{3 \mathrm{a}}[\mathrm{Ni}]_{3 \mathrm{~b}}\left[\mathrm{O}_{2}\right]_{6 \mathrm{c}}$ true formula. This results in a lower initial capacity as well as severe capacity loss upon cycling. Co substitution for Ni leads to strictly two-dimensional $\mathrm{LiNi}_{1-y} \mathrm{Co}_{\mathrm{y}} \mathrm{O}_{2}$ oxide without extra-nickel in the lithium plane. We have selected the $\mathrm{LiNi}_{0.7} \mathrm{Co}_{0.3} \mathrm{O}_{2}$ composition for the electrochemical study. In order to increase its electronic conductivity and to make easier the lithium diffusion during the intercalation process, two ways were chosen: i) synthesis by the combustion method which leads to small particle sizes, ii) substitution of $\mathrm{Mn}$ for $\mathrm{Ni}$ and $\mathrm{Co}$, which leads to the existence of mixed valence system. The results of structural study were discussed in relation with magnetic and electrochemical properties.

On the other hand, $\mathrm{LiMn}_{2} \mathrm{O}_{4}$ adopts the spinel structure

(space group $\mathrm{Fd} \overline{3} \mathrm{~m}$ ) consisting of a cubic close packed oxide ions with the manganese ions in one half of the octahedral site (16d wyckoff site) and $\mathrm{Li}^{+}$ions in oneeighth of tetrahedral sites (8a site). Although of its low coast and good environmental benignity, this oxide suffers from a serious capacity deterioration problem after cycling at high temperatures which seems to be related to the existence of Jahn-Teller $\mathrm{Mn}^{3+}$ ions. In order to improve the rate capability of the materials and to keep the oxidation state of manganese as $\mathrm{Mn}^{4+}$, we have carried out the synthesis of nanocrystalline double substituted spinels $\mathrm{LiNi}_{1.5-\mathrm{y}} \mathrm{Ni}_{0.5-\mathrm{y}} \mathrm{Cr}_{2 \mathrm{y}} \mathrm{O}_{4}$. The samples have been characterized at first by X-ray diffraction. The Scherrer method has been used to elucidate the thermal evolution of the average crystallite sizes. The relationship between the structural data and the electrochemical performances of the studied samples will be presented during the ECM meeting.

Acknowledgements: Many thanks to the French Foreign Ministry (MAE) for the financial support under the Corus program (contract no 02211 121)

\section{MS17 O4}

High-pressure structural studies of energetic compounds C. R. Pulham ${ }^{a}$ A. J. Davidson, ${ }^{a}$ I. D. H. Oswald, ${ }^{\text {a }}$ D. I. A. Millar, ${ }^{a}$ F. P. A. Fabbiani, ${ }^{\text {b }}$ D. J. Francis, ${ }^{\mathrm{b}}$ W. G. Marshall, ${ }^{\mathrm{b}}$ A. S. Cumming, ${ }^{\mathrm{c}}$ D. R. Allan, ${ }^{\mathrm{d}}$ A. R. Lennie ${ }^{\mathrm{e}}$ and T. J. Prior. ${ }^{\mathrm{e}}{ }^{\mathrm{a}}$ School of Chemistry and Centre for Science at Extreme Conditions, University of Edinburgh,UK. ' ${ }^{\mathrm{b}} I S I S$ Neutron Facility, Rutherford Appleton Laboratory,UK. ${ }^{\mathrm{c} D s t l,}$ Fort Halstead, Sevenoaks,UK. ${ }^{\mathrm{d}}$ Diamond Light Source, UK. ${ }^{e}$ SRS, Daresbury Laboratory, Warrington, UK

E-mail: C.R.Pulham@ed.ac.uk

Keywords: energetic materials, high-pressure diffraction, polymorphism

The effective modelling of the properties and performance of solid energetic materials (propellants and explosives) under operational conditions requires detailed knowledge of the crystal structures of these compounds. In many cases, the crystal structure of the compound obtained under ambient conditions is used as the basis for modelling properties at higher temperatures and pressures because structural information is often not available under more extreme conditions. However, such extreme conditions can lead to substantial changes in intermolecular interactions and molecular geometries, and can even induce phase transitions leading to dramatic changes in properties such as detonation velocity, shock sensitivity, and reactivity. There is therefore a requirement to obtain detailed structural information about these materials under extreme conditions. Using a combination of X-ray and neutron diffraction techniques, augmented by vibrational spectroscopy, we have studied a series of energetic ammonium salts and have obtained detailed structural information for these materials at pressures up to $\sim 8 \mathrm{GPa}$. Under hydrostatic conditions ammonium perchlorate undergoes a first order phase transition at $3.98 \mathrm{GPa}$ in agreement with the results of a previous study [1]. We have successfully solved and refined the structure of this new orthorhombic phase, which features a more close-packed structure with more extensive hydrogen bonding. Contrary to a previous report [2], no evidence was found for a pressure-induced phase transition in ammonium dinitramide up to $4.03 \mathrm{GPa}$ - an equation of state up to this pressure has been obtained. Significant changes were observed in the intermolecular contacts involving N-H...O-N interactions, and in the torsional angles of the dinitramide ion. We have also studied the effects of pressure on the widely used secondary explosives HMX and RDX. Structural information on the $\beta$-form of HMX up to $\sim 8 \mathrm{GPa}$ has been obtained. Neutron powder diffraction studies of RDX up to $\sim 8 \mathrm{GPa}$ confirm the previously observed $\alpha-\gamma$ transition at $\sim 4 \mathrm{GPa}[3,4]$ and suggest a significant conformational change in the high-pressure polymorph. 